\title{
LASIK em pacientes adultos com alta anisometropia: relato de casos
}

\author{
LASIK for high anisometropia in adults: case report
}

\author{
Patrícia Ioschpe Gus ${ }^{1}$ \\ Guilherme Herrmann Matos ${ }^{2}$ \\ Marcia Cristina Bayer ${ }^{3}$
}

Trabalho realizado na Disciplina de Sistema Visual da Faculdade Medicina da Universidade Luterana do Brasil.

${ }^{1}$ Doutora em Oftalmologia e Professora da Disciplina de Sistema Visual da Faculdade de Medicina na Universidade Luterana do Brasil - ULBRA - Porto Alegre (RS) - Brasil.

${ }^{2}$ Acadêmico do $11^{\circ}$ semestre da Faculdade de Medicina da - ULBRA - Porto Alegre (RS) - Brasil.

${ }^{3}$ Médica formada pela Faculdade de Medicina da ULBRA - Porto Alegre (RS) - Brasil.

Endereço para correspondência: Guilherme Herrmann Matos - Rua Comendador Castro, 515 - Porto Alegre (RS) CEP 91760-200

E-mail: matos81@bol.com.br ou ghmatos@globo.com

Recebido para publicação em 20.02.2004

Versão revisada recebida em 21.07.2005

Aprovação em 02.12.2005

Nota Editorial: Depois de concluída a análise do artigo sob sigilo editorial e com a anuência da Dra. Núbia Cristina F. Maia sobre a divulgação de seu nome como revisora, agradecemos sua participação neste processo.

\section{RESUMO}

Objetivo: Avaliar a eficácia da técnica laser in situ keratomileusis (LASIK) na redução de anisometropia em adultos, para os quais os tratamentos convencionais não tiveram sucesso. Métodos: Série de casos de três olhos de três pacientes adultos, dois do sexo feminino e um do sexo masculino, com idade entre 28 e 49 anos (média de 38,3 anos), os quais foram submetidos à técnica de LASIK. Dois pacientes foram acompanhados por dezoito meses e um por seis meses após a cirurgia. Resultados: Comparando a acuidade visual corrigida do pré-operatório com a acuidade visual não corrigida do pós-operatório, um olho ganhou duas linhas de visão, um olho manteve-se igual e um olho perdeu uma linha de visão. Todos os olhos se mantiveram sem ametropia esférica, e o astigmatismo não excedeu -0,75 D no pós-operatório. Conclusão: A técnica LASIK mostrou-se eficaz na correção de alta anisometropia em adultos, melhorando a acuidade visual e as queixas astenopéicas, e diminuindo a anisometropia.

Descritores: Ambliopia, Anisometropia/cirurgia; Ceratomileuse assistida por excimer laser in situ/métodos; Erros de refração/cirurgia; Acuidade visual; Relatos de casos [tipo de publicação]

\section{INTRODUÇÃ̃O}

A anisometropia é definida como a assimetria de erro refracional entre os dois olhos, podendo existir quando a diferença esférica ou cilíndrica for igual ou superior a 1,00 dioptria(D) na ausência de estrabismo ${ }^{(1)}$. Quando superior a 3,00 D surgem sintomas de astenopia como ardência, prurido, diplopia, cefaléia, lacrimejamento, fotofobia e visão borrada ${ }^{(2-6)}$. Esses sintomas são causados pela aniseicônia, que corresponde à diferença na formação do tamanho das imagens retinianas entre os dois olhos e pelo deslocamento prismático das imagens causados pela correção com óculos ${ }^{(3)}$.

Os tratamentos da ambliopia anisometrópica incluem correção do erro refracional com óculos ou lentes de contato, e oclusão temporária do olho dominante em crianças. Algumas vezes, a correção completa com óculos não é possível, sendo necessária a diminuição do grau do olho amblíope para amenizar a aniseiconia, ou o uso de lentes de contato. Entretanto, parte dos amblíopes anisométropes não se adaptam com a hipocorreção, nem com o uso das lentes. Para esses pacientes, a cirurgia refrativa aparece como uma alternativa viável ${ }^{(2-8)}$.

Alguns estudos mostram ganho de uma a duas linhas na acuidade visual não corrigida de crianças e adolescentes com anisometropia após a realização da cirurgia refrativa com os métodos de PRK ou LASIK ${ }^{(2-8)}$. Entretanto, existem poucos relatos mostrando resultados da cirurgia refrativa em pacientes adultos com anisometropia. Nesse estudo avaliamos a eficácia do LASIK para alta anisometropia em 3 pacientes adultos. 


\section{MÉTODOS}

Em um estudo prospectivo, três olhos de três pacientes, dois do sexo feminino e um do sexo masculino, com idade entre 28 e 49 anos (média de 38,3 anos), foram submetidos à técnica LASIK para correção de alto erro refracional em um dos olhos de cada paciente. Todos os pacientes apresentavam anisometropia e dois apresentavam ambliopia.

Todos os pacientes realizaram avaliação pré-operatória através de refração estática e dinâmica (Topcon), biomicroscopia (Topcon), oftalmoscopia indireta binocular (Welch Allyn), tonometria (Goldman), ceratometria (Storz), topografia corneana (Storz) e paquimetria ultra-sônica (Storz), e responderam questionário relativo a queixas de astenopia antes e após a cirurgia.

As cirurgias foram realizadas sob anestesia tópica com o excimer laser Ladarvision 4000 e o microcerátomo SKBM. Os três olhos foram operados pela mesma cirurgiã. Nenhum paciente apresentou complicações transoperatórios.

Os critérios para medir a eficácia no pós-operatório foram: diminuição da anisometropia, melhora da acuidade visual não corrigida do olho operado e melhora da visão binocular com diminuição da astenopia. Dois pacientes foram acompanhados por 18 meses e um por seis meses após a cirurgia.

\section{RESULTADOS}

Comparando a acuidade visual corrigida do pré-operatório com a acuidade visual não corrigida do pós-operatório, um olho ganhou duas linhas de visão, um olho manteve-se igual e um olho perdeu uma linha de visão, conforme tabelas 1 e 2 . Todos os olhos amblíopes tiveram melhora em sua acuidade visual com e sem correção. Os três olhos se mantiveram sem grau esférico, e o astigmatismo não excedeu $-0,75 \mathrm{D}$ no pósoperatório em um caso, conforme tabela 2. Houve melhora dos sintomas de astenopia nos três pacientes.

\section{DISCUSS ÃO}

A cirurgia refrativa com excimer laser para correção de miopia, astigmatismo e hipermetropia, tem sido utilizada na população adulta com sucesso. A experiência dos médicos que utilizam excimer laser aumenta a cada dia, e da mesma forma as indicações para seu uso. A cirurgia refrativa para anisométropes poderá tornar-se uma indicação, baseado nos resultados positivos encontrados por alguns autores ${ }^{(1-8)}$.

Sabe-se que anisometropia de até 3,00 D pode ser tolerada pela maioria dos anisométropes com os tratamentos convencionais. Sendo assim, pode-se recomendar a cirurgia em pacientes com diferença de 3,00 a $8,00 \mathrm{D}$. Os métodos de PRK e LASIK demonstram promissores resultados em pacientes com alta miopia anisometrópica ${ }^{(7)}$. A maioria dos estudos atuais são realizados em pacientes jovens, entre 6 e $15 \operatorname{anos}^{(3-6)}$.

Barbosa et al. analisaram a eficácia e a segurança do LASIK em 27 olhos de 15 pacientes adultos (média de idade de 29 anos) com anisometropia maior ou igual a 2,50 D. A média do equivalente esférico antes da cirurgia era de $-6,64 \mathrm{D}$, e da anisometropia 5,85 D. Após a cirurgia, a anisometropia média baixou para $1,11 \mathrm{D}$. A acuidade visual sem correção melhorou em 26 olhos e manteve-se inalterada em um, com ganho de uma a doze linhas de visão. Nove dos dez pacientes que apresentavam astenopia relataram melhora dos sintomas ${ }^{(7)}$.

Alguns autores, em estudo retrospectivo, avaliaram a segurança e eficácia do uso do PRK para correção de alta miopia em criança de 7 a 15 anos com anisometropia. A média do equivalente esférico antes da cirurgia era de -8,93 D, e após, de $-1,66$ D. Nenhum olho perdeu linhas de visão, nove olhos ganharam uma linha e cinco ganharam duas linhas ${ }^{(6)}$.

Outros autores avaliaram 14 olhos de 14 crianças (entre $7 \mathrm{e}$ 15 anos) após LASIK no olho com maior miopia. A média do equivalente esférico antes da cirurgia era de $-7,87 \mathrm{D}$, e a média da acuidade visual com correção era de 20/50. Um ano após a cirurgia a média do equivalente esférico baixou para $-0,55 \mathrm{D}$, e

\begin{tabular}{|lccccccc|}
\hline Nome & Sexo & Idade & $\begin{array}{c}\text { Ano da } \\
\text { cirurgia }\end{array}$ & $\begin{array}{c}\text { Olho } \\
\text { operado }\end{array}$ & $\begin{array}{c}\text { Refração estática } \\
\text { do olho operado }\end{array}$ & $\begin{array}{c}\text { Refração estática } \\
\text { do olho adelfo }\end{array}$ & $\begin{array}{c}\text { AVCC pré-operatória } \\
\text { do olho operado }\end{array}$ \\
S.S.S. & M & 38 & 2003 & OE & $+6,50$ plano & $+0,50$ plano & $20 / 150$ \\
F.F.M. & $F$ & 28 & 2003 & OD & $+1,00-5,00 \times 180^{\circ}$ & $+1,50-1,25 \times 180^{\circ}$ & $20 / 80$ \\
S.D.Z. & $F$ & 49 & 2002 & OE & Plano $-4,00 \times 180^{\circ}$ & plano $-1,00 \times 5^{\circ}$ & $20 / 20$ \\
M = Masculino; F = Feminino; OD = Olho direito; OE = Olho esquerdo; AVCC = acuidade visual com correção & \\
\hline
\end{tabular}

\begin{tabular}{|lcccc|}
\hline & \multicolumn{2}{c|}{ Tabela 2. Refração estática e acuidade visual sem correção Pós-LASIK } \\
Nome & $\begin{array}{l}\text { Refração estática } \\
\text { do olho operado }\end{array}$ & $\mathbf{2 4}$ horas & Uma semana & Seis meses \\
S.S.S. & Plano & $20 / 70$ & $20 / 60 \mathrm{p}$ & Não retornou \\
F.F.M & Plano & $20 / 40$ & $20 / 40$ & $20 / 60 \mathrm{p}$ \\
S.D.Z. & Plano $-0,75 \times 180^{\circ}$ & $20 / 15 \mathrm{p}$ & $20 / 20$ & $20 / 20$ \\
P parcial & & & & Não retornou \\
\hline
\end{tabular}


a média da acuidade visual com correção mudou para 20/25, e sem correção para 20/30. Todos os pacientes tiveram ganho de no mínimo duas linhas de visão ${ }^{(5)}$.

Alguns autores acompanharam, durante três anos, nove olhos de nove crianças, com idade entre 8 e 15 anos, após terem sido submetidas ao LASIK para correção de alta anisometropia. Após três anos, todos os pacientes tiveram ganho de no mínimo cinco linhas de visão e 55,5\% apresentavam acuidade visual sem correção de 20/50 ou melhor. A média do equivalente esférico pré-operatório foi reduzido de -7,66 D para 1,02 $\mathrm{D}^{(3)}$.

A maioria dos oftalmologistas utilizam como critério para quantificar o sucesso da cirurgia refrativa a permanência de grau residual entre -1,00 D e +1,00 D. Entretanto, pensamos que em pacientes anisométropes, a simples diminuição do grau para níveis inferiores a 2,5 D já possibilita melhor visão binocular e maior conforto visual ${ }^{(7)}$. Por isso, nessa série de casos, pacientes que tiveram discreta melhora da acuidade visual foram considerados como bem sucedidos na cirurgia.

\section{CONCLUSÃO}

Os presentes casos indicam que o procedimento pode ser efetivo e previsível. A correção cirúrgica do olho com maior erro refracional pode ser benéfica, pois melhora subjetivamente a qualidade de vida dos pacientes com olhos anisométropes.

\section{ABSTRACT}

Purpose: To evaluate the efficacy of the laser in situ keratomileusis (LASIK) technique in the reduction of anisometropy in adults, for whom conventional treatment was not successful. Methods: A sequence of cases of three eyes of three adult patients, two females and one male, age ranging from 28 and 49 years (average 38.3 years), who underwent LASIK. Two patients were followed up for eighteen months and one for six months. Results: Comparing the corrected visual acuity before surgery with the non-corrected visual acuity after surgery, one eye gained two lines of vision, one eye remained the same and one eye lost one line of vision. All eyes remained without spheric refraction, and astigmatism did not exceed $-0.75 \mathrm{D}$ after surgery. There has been an improvement in the symptoms of asthenopia in the three patients. Conclusion: The LASIK technique proved to be efficient in the correction of anisometropy in adults, improving visual accuity and asthenopia complaints, and leading to a similar refraction in both eyes.

Keywords: Amblyopia: Anisometropia/surgery; Keratomileusis, laser in situ/methods; Refractive errors/surgery; Visual acuity; Case reports [publication type]

\section{REFERÊNCIAS}

1. Attebo K, Mitchell P, Cumming R, Smith W, Jolly N, Sparkes R. Prevalence and causes of amblyopia in adult population. Ophthalmology. 1998;105(1): 154-8.

2. Maden A, Erkin EF, Oner FH. Unilateral refractive keratotomy for anisometropia. J Refract Surg. 1998;14(3):325-30.

3. Nassaralla BRA, Nassaralla Junior JJ. Três anos pós-lasik em crianças anisométropes de 8 a 15 anos de idade. Arq Bras Oftalmol. 2003;66(2):145-51.

4. Astle WF, Huang PT, Ells AL, Cox RG, Deschenes Mc, Vibert HM. Photorefractive keratectomy in children. J Cataract Refract Surg. 2002;28(6): 932-40.

5. Rashad KM. Laser in situ keratomileusis for myopic anisometropia in children. J Refractive Surg. 1999;15(4):429-35.

6. Autrata R, Rehurek J. Clinical results of excimer laser photorefractive keratectomy for high myopic anisometropia in children: four-year follow-up. J Cataract Refract Surg. 2003;29(4):694-702.

7. Barbosa R, Nassaralla B. Lasik para anisometropia associada à ambliopia em pacientes adultos. Rev Bras Oftalmol. 2001;60(2):97-103.

8. Bilgihan K, Özdek S, Gürelik G, Adigüzel U, Önol M, Hasanreisoglu B. Photorefractive keratectomy visual rehabilitation of anisometropia induced by retinal detachment surgery. J Refractive Surg. 2000;16(1):75-8. 